\title{
Evaluación financiera de plantaciones forestales comerciales
}

\author{
Pedro Ernesto del Castillo Cueva ${ }^{1}$
}

\section{RESUMEN}

\begin{abstract}
Las actividades de reforestación en México no han rendido frutos conmensurables con el esfuerzo y recursos invertidos en ellas. Un problema fundamental es que se olvida que los terrenos tienen dueño, incluso los de propiedad pública; por lo tanto, a las plantaciones no se les ha dado el seguimiento requerido. El éxito de los programas actuales en el Estado de Veracruz se mide por el porcentaje de prendimiento en el campo y no por el número de plantas que salen de los viveros. Se tiene en la entidad un programa de apoyo a productores, para el cual se pretende conseguir la participación de la banca nacional. Para ello se presenta información de tipo financiero (costo/ha de plantaciones), así como los dos tipos de apoyo financiero para productores de acuerdo con su nivel de ingresos. Se hace una amplia discusión acerca de la rentabilidad de las plantaciones y se comentan los aspectos necesarios para crear una cultura silvícola a nivel estatal.
\end{abstract}

\section{PALABRAS CLAVE}

Reforestación, plantaciones comerciales, viveros, análisis financieros, rentabilidad, cultura silvícola.

\begin{abstract}
Reforestation activities in Mexico have not met with success that, given the large amount of efforts and resources spent, deserves. A fundamental problem is that the land has owners, whether private or public, and the plantations have not received the required follow-up. The success of current programs in the State of Veracruz is measured by the percentage of surviving plants in the field, instead of by the number of plants that come out of nurseries. The government of the State of Veracruz has established a program to support tree producers and economic participation by financial institutions is being sought. Financial information is presented (cost/hectare of tree plantations), as well as the two types of financial support available to producers according to their income levels. The feasibility of plantations and the required elements to create a forestry culture at the state level are throughly discussed.
\end{abstract}

\section{KEY WORDS}

Reforestation, commercial plantations, nurseries, financial analysis, forestry culture.

1 Secretario de desarrollo Agropecuario, Forestal y Pesquero. Gobierno del estado de Veracruz; Presidente de la Asociación Mexicana de Secretarios de Desarrollo Agropecuario, A.C. J.J. Herrera No. 691000 Xalapa, Ver. México. Manuscrito recibido para su publicación el 21 de julio de 1995. 


\section{INTRODUCCION}

Durante mucho tiempo en México se ha debatido sobre la necesidad de reforestar, de ganarle en velocidad a la deforestación, es decir, reforestar más de lo que se deforesta anualmente. Se ha hecho esfuerzos inusitados y tal vez, se ha gastado demasiado dinero en tratar de desarrollar programas de reforestación en nuestro país $y$, es de lamentar que prácticamente no existan evidencias para conocer ese avance o los resultados concretos. Es decir, poco es lo que se puede certificar en el campo que sea compatible con el esfuerzo, la voluntad, con el interés de la sociedad y con el dinero que se ha depositado en esto.

Pensamos que el principal problema estriba en que se ha olvidado un elemento fundamental: que los terrenos donde se reforesta tienen dueño, y que esos terrenos, al tener dueño, pasan por un proceso de costo por oportunidad: entre dejarlos en la reforestación o dedicarlos a otras cosas, y siempre prevalece la razón económica. Por eso mucha de la reforestación que se hace, los propios dueños de los bosques se encargan de que desaparezca porque toca un aspecto que tiene que ver con su bolsillo y con su modo de vida. Creemos que reconocer que cada terreno forestal tiene dueño y que ese dueño necesita encontrar en la reforestación una opción económica, es el cambio que se busca en Veracruz, sin dejar de reconocer que existen terrenos que no son de nadie en particular por ser áreas que pertenecen a la Federación, a un estado, a la reserva de un municipio, o por ser un área ecológica pero, en este caso los dueños están representados por los gobiernos respectivos. El problema, la deformación en nuestra sociedad, en nuestro concepto, es que se piensa que los terrenos baldíos son tierra de nadie y que se puede hacer ahí lo que se quiera, que basta con llevar una planta y ponerla ahí para que se vuelva un bosque dentro de poco, sin tomar en cuenta que una planta requiere un tratamiento, un seguimiento, una atención, un manejo y un permanente cuidado.

Todas estas contradicciones nos han llevado a tirar dinero en forma de arbolitos. Para dar una idea, en los últimos cuatro años, antes de 1992, salieron de viveros 136 millones de plantas que serían suficientes para sembrar entre 50 y 60 mil hectáreas en Veracruz, sin embargo, en toda la historia de Veracruz, incluyendo los programas de reforestación de los gobiernos estatales más recientes no hemos contabilizado más de 3 mil hectáreas reforestadas.

\section{PROGRAMAS DE REFORESTACION VIGENTES}

A petición del titular del Gobierno Estatal, se le da un carácter serio a la reforestación y se están midiendo los resultados por el número de plantas que se logran en campo y no por el número de plantas que producen en vivero. Ese es el cambio que se busca en Veracruz, ¿cómo lograr que alguien que tiene un terreno con potencial, que afortunadamente tenemos en Veracruz pueda encontrarle atractivo a la reforestación?, ¿cómo despertar el interés de los propietarios para que esa plantación se convierta en un negocio viable, para que realmente veamos un bosque a futuro como recomiendan las normas internacionales?, y para que el porcentaje de prendimiento en el campo efectivamente esté en $70 \mathrm{u}$ 80 por ciento y no el 9 ó 10 por ciento que históricamente se ha logrado en México.

Es preciso que reconocer primero que los terrenos boscosos tienen dueño, que la decisión final de sembrar árboles o no 
debe ser del propio productor, que mal haríamos en pedirle que nos dejara meter unas plantas para reforestar su terreno aunque le paguemos, porque él se va ha encargar de que esas plantas se quiten; entonces, para interesarlo necesitamos hacerle un planteamiento de tipo económico. Vamos a acotar el asunto, vamos a seleccionar las áreas en donde el interés público tiene que prevalecer, en donde se tienen que hacer los proyectos de reforestación y de rescate tal como se hace en el Parque Nacional con el programa de recarga de acuíferos a través de la construcción de tinas ciegas, con las presas filtrantes, etc., esto es de interés público y eso sí es responsabilidad nuestra hacerlo.

Tenemos que reforestar las cuencas hidrográficas de forma permanente sobre todas las cabeceras, tenemos que convenir con los municipios y ya lo estamos haciendo con Xalapa, para rescatar todas esas cuencas, tenemos que hacerlo también con Coatzacoalcos, ahí por una razón ecológica, es una razón de preservación, es decir, no es una razón económica. Hay un punto de confluencia, hay ocasiones en las que un terreno puede cumplir un objetivo ecológico y también, al mismo tiempo un objetivo económico, pueden darse ambos casos. Cuando eso sucede hay una mezcla de recursos en donde el gobierno aporta un porcentaje y el particular, otra proporción.

Se debe demostrar que la actividad forestal puede ser una buena opción, que puede ser rentable y en nuestro estado sabemos que si hay posibilidad de hacer rentable una plantación, es decir, con los crecimientos que tenemos en Veracruz, por ejemplo en la región Noreste del Cofre de Perote se pueden obtener dos millones y medio de nuevos pesos de ingreso por hectárea en una plantación forestal. Esta estimación se basa en que los crecimientos anuales son de 20 y 25 metros cúbicos, esa es una razón económica. Se ha trabajado mucho para que se conozca que las plantaciones tienen que considerarse de la misma forma que una milpa o siembra de aguacate o de manzanas, que se tienen que cuidar, que cercar, que podar y que atender; lo mismo tenemos que hacer con plantación y no lo hemos hechos. La recuperación de la inversión en materia forestal es de largo plazo, no es precisamente atractivo estar cuidando un arbolito que va a dar los primeros resultados en quince años, no es fácil esperar a que los dueños, en general descapitalizados, destinen casi toda su tierra a un cultivo a tan largo plazo.

\section{ESTRATEGIAS DE FINANCIAMIENTO}

La nuestra es una sociedad descapitalizada y debemos buscar cómo lograr que se cultiven los bosques. La primera acción a realizar es localizar, saber que este terreno tiene dueño, que al dueño lo vamos a convencer de que puede obtener beneficios económicos ahí y una vez convencido, plantearle que su proyecto es rentable. Si hay un problema de rentabilidad, porque las condiciones del suelo no sean las adecuadas y de todas maneras resulta de interés público reforestar ahí, el gobierno tiene que aportar recursos dentro de un esquema de financiamiento, aligerando el costo financiero del proyecto y dándole viabilidad económica. En suma, primero, tenemos que reconocer que el productor es el dueño de la tierra; segundo, que él toma la decisión; tercero, demostrarle que la plantación es un negocio rentable y; cuarto, si no es rentable el sector oficial debe aportar lo necesario para hacerlo viable, no para el gobierno, sino para el productor (Fig. 1). 


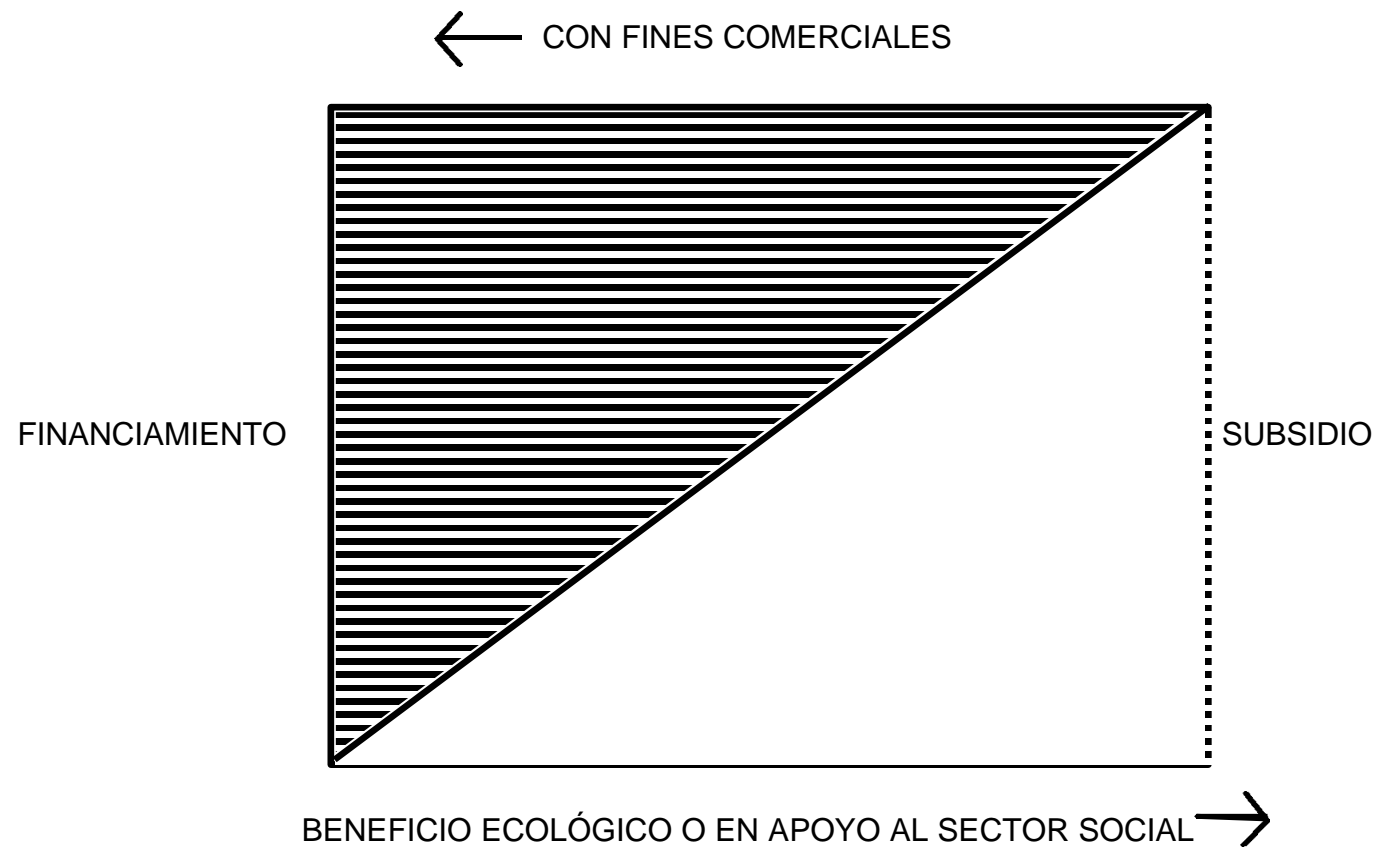

Figura 1. Interrelación financiamiento / beneficio

Una vez que esté convencido que la actividad es rentable, que se decida a sembrar, después de escoger las especies adecuadas, tenemos que convencer al banco de ésta rentabilidad, de que es posible hacer un negocio en el área forestal. Convencido el banco, se cuenta con el dinero para que esté productor sobreviva en el período preproductivo y le damos la posibilidad de hacer viable esa plantación. Por eso es tan importante que participen los promotores, los técnicos, y los banqueros, para que hablemos el mismo lenguaje y que sepamos como evaluar la rentabilidad de una plantación. Hay métodos y técnicas por computadora ya probados y también hemos realizado los análisis financieros y estamos convencidos de que es posible que los proyectos forestales son rentables o los podemos hacer rentables sumando los recursos del gobierno, así evitamos tirar dinero público en forma de arbolitos.

\section{RENTABILIDAD DE LAS PLANTACIONES}

Es muy importante señalar en primer lugar que hay métodos para evaluar la rentabilidad de las plantaciones. Si se sabe como evaluar una plantación, se puede calcular su tasa interna de retorno y presentarse al banco con estos argumentos en busca de apoyo financiero para un proyecto. Si la tasa interna de retorno calculada para la plantación siendo evaluada está por debajo de los requerimientos del banco, es responsabilidad del gobierno, si existe el interés por reforestar, plantear un posible acuerdo con la banca para que, entre ambos, lograr la tasa interna de retorno deseada. Es aquí donde desempeña un papel fundamental el apoyo estatal al proporcionar la planta, la cual de cualquier manera se suministra. Muchos proyectos van a ser viables desde el principio, pero muchos otros no, pero desde el punto de vista del gobierno estatal todos tienen que 
ser viables; es decir, si la rentabilidad no es adecuada, el gobierno del estado ofrece la posibilidad de ir ajustando esa tasa. Si con el suministro de la planta todavía no se logra la rentabilidad deseada, se ofrecerá el apoyo adicional del cercado, pues el objetivo final es fomentar la reforestación en suficiente número de predios. En estas condiciones se calcula nuevamente la taza interna de retorno. Si no es suficiente todavía se ofrecerán apoyos como despensas para pagar la apertura de cepas y así sucesivamente hasta que se logre el objetivo de hacer atractivo para el productor sembrar árboles y cuidarlos.

En el caso de que los terrenos elegidos tengan más bien un carácter ecológico, o sean terrenos no aptos para fines comerciales, entonces las dependencias que tendrían que hacer la labor de reforestación son la Dirección General de Asuntos Ecológicos del Estado o la Delegación estatal de la secretaría del ramo (SEMARNAP), o la misma oficina de la SEDAP, pero no se puede pretender la participación de la banca porque ese proyecto no es viable desde el punto de vista comercial.

Es necesario enfatizar estos conceptos entre la banca nacional toda vez que hay una gran variedad de casos, desde los proyectos que son rentables por tratarse de áreas bien escogidas y ser zonas adecuadas para plantaciones, hasta aquellos donde el crecimiento va a ser muy lento y no existen posibilidades de sostenerlo como proyecto financiero. El Gobierno del Estado tiene fundadas esperanzas en estos esquemas de financiamiento; sostenemos que las plantaciones en Veracruz va a ser viables en la medida en que se tenga un seguimiento y una forma de hacerles llegar a los productores recursos para hacer un trabajo serio de silvicultura en las plantaciones. En el estado hemos tenido mucho éxito, las plantaciones que se han financiado hasta ahora, llevan buen desarrollo, han sido detonadores para que las instituciones financieras como el fideicomiso en relación a la agricultura (FIRA) y otros bancos empiecen a ver en esto posibilidades de negocio. El éxito de las plantaciones de cedro y primavera en la zona sur están dando muy buenos elementos, lo mismo sucede con las plantaciones de hule.

Quisiera que existiera un criterio único, de manera que al presentarse proyectos de financiamiento para plantaciones, la banca entienda bien el proyecto para evitar frustraciones. Se han dado casos de despachos especializados en elaborar proyectos de financiamiento forestal que al llegar al banco, se encuentran con que los funcionarios encargados desconocen si es posible otorgar el financiamiento 0 no, que si se va a dar la tasa cero, situación que desanima a los productores interesados en hacer un trato con el banco. Es necesario aumentar la comunicación con los banqueros porque sentimos que la banca puede hacer un buen negocio y puede llenar a Veracruz de bosques tropicales y de clima templado, fundamentado en un aspecto de interés por parte del dueño y un aspecto de economía que no solamente va a ser significativo no sólo para él, sino para la entidad.

Con estos razonamientos extiendo una invitación para seguir impulsando estos programas. Estamos conscientes de que no es un asunto sencillo. El proyecto de financiamiento forestal que funciona en la actualidad es el esquema de financiamiento FIRA que nos tocó iniciar en la anterior Administración Federal. Trabajamos mucho con FIRA en diferentes 
partes del país para llegar al esquema de esté utilizando muy poco porque la banca aun no participa en forma amplía, dado que no cuenta con el personal que sepa evaluar plantaciones por tratarse de algo nuevo. Estamos seguros de que está si es una opción viable.

\section{CONCLUSIONES}

Si alguna explicación tiene el por qué se han destruido los bosques es porque se han destruido tierras forestales o de vocación forestal al crearse el Banco Agrícola orientado a dar créditos agrícolas y ganaderos y áreas forestales. Ese fue el detonador y, la consecuencia que tuvo el dar créditos agrícolas y ganaderos en zonas forestales, fue derribar los árboles creando áreas muy deforestadas. Por eso el crédito forestal da muchas opciones; primero, dar un tratamiento a las áreas forestales haciéndolas efectivamente viables; segundo, crear un nuevo tipo de productores que sean silvicultores, porque hay agricultores y ganaderos pero no silvicultores y una buena parte de nuestra superficie es silvícola, son bosques, son selvas. Es necesario crear la clase de silvicultores y así como se sustentan los ganaderos, como se sustentan los agricultores, así se tiene que organizar este grupo, porque hasta ahora solamente han sido extractores, cortando los árboles y extrayéndolos, pero no han cultivado el bosque. Esto equivale a estar en la etapa de la recolección, como si estuviéramos todavía en el neolítico, es decir, se sigue recolectando, como se recolectan peces. Se tiene que hacer acuacultura y silvicultura, cultivar, que el esfuerzo del hombre oriente la financiamiento actual. Lamentamos que se producción. Para hacer silvicultura se necesita dinero, dinero con un periodo de recuperación también especial ad hoc para la condición forestal. La meta ahora es seguir avanzando con el apoyo de la banca y de los técnicos especializados cambiando proyectos de reforestación a proyectos de plantación. La reforestación se va a ser en la áreas con un sentido ecológico estrictamente. A la Secretaria de Desarrollo Agropecuario, Forestal y Pesquero le corresponde la parte productiva, es en lo que estamos esforzándonos.

Sabemos que está gestando un cambio de fondo en cada uno de los que formamos parte de este programa. En ninguna parte de México se está luchando con tanto empeño en esté sentido, es decir, en materia de créditos forestales para cultivar bosques. Se han hecho proyectos grandes en otras partes; en el Estado de Veracruz nos estamos abocando a los pequeños y tenemos una respuesta copiosa. Desde Pánuco hasta las Choapas, se empiezan a ver plantaciones de seda o de melina, que antes no existían. Es gratificante ver que muchos de ellos cuentan con el respaldo de la banca. Estamos seguros que vamos por el camino adecuado, dando una opción a la sociedad, que es darle un sentido serio, profesional, de honestidad al tema de la reforestación, fundamentando en cosas reales que se traduzcan en árboles, en recuperación de áreas. Realmente estamos tocando el punto más sensible que es el financiamiento. 